\title{
The Pharmacological Mechanism of the Effect of Plant Extract Compound Drugs on Cancer Pain Based on Network Pharmacology
}

\author{
Yuanyuan Shen, Jun Wang, Pengpeng Yan, Tiantian Chen, Xingrui Li, and Ming Jiang \\ Department of Anesthesia, Luoyang Hospital of TCM, Luoyang 471000, China \\ Correspondence should be addressed to Ming Jiang; he_xin_chen_123@163.com
}

Received 17 November 2021; Revised 7 January 2022; Accepted 12 January 2022; Published 27 February 2022

Academic Editor: Bhagyaveni M.A

Copyright (c) 2022 Yuanyuan Shen et al. This is an open access article distributed under the Creative Commons Attribution License, which permits unrestricted use, distribution, and reproduction in any medium, provided the original work is properly cited.

\begin{abstract}
Objective. We systematically analyzed the mechanism of plant-derived drugs alleviating cancer pain in our hospital through network pharmacology, so as to provide the possibility of further application of traditional Chinese medicine in the treatment of cancer pain. Methods. We used TCMSP, ETCM, and TCMID databases to mine the active ingredients of plant-derived drugs. We combined OMIM, GeneCards, and DrugBank databases to mine and match the common targets of plant-derived drugs for cancer pain. We used the STRING platform and Cytoscape software to analyze and screen out the core targets. We used GO and KEGG methods to analyze the biological processes, molecular functions, cellular composition, and signaling pathways involved in the reduction of cancer pain by plant-derived drugs. Results. We found 153 active ingredients from botanical drugs by TCMSP (Traditional Chinese Medicine Systems Pharmacology Database and Analysis Platform, TCMSP), ETCM (The Encyclopedia of Traditional Chinese Medicine), and TCMID (Traditional Chinese Medicine Integrated Database) databases, covering 341 protein targets in human body. Combined with OMIM (Online Mendelian Inheritance in Man), GeneCards, and DrugBank databases, we excavated and matched 141 targets of plant-derived drugs and cancerous pain diseases. Through the analysis of the STRING platform and Cytoscape software, 19 core targets including TNF, MAPK1, JUN, and IL-6 were screened out. Go and KEGG enrichment showed that plant-derived drugs alleviated cancer pain processes involving 193 biological processes, 47 molecular functions, 22 cell components, and 118 signaling pathways. By screening genes involved in KEGG signaling pathway, it was found that plant-derived drugs were mainly associated with PI3K-Akt signaling pathway, tumor necrosis factor signaling pathway, MAPK signaling pathway, Toll-like receptor signaling pathway, and HIF-1 signaling pathway in alleviating cancer pain. Conclusion. These results indicate that botanical drugs can positively affect the expression of inflammatory factors and apoptotic factors in the process of treatment and relief of cancer pain, which is expected to have a potential therapeutic effect on the relief of cancer pain.
\end{abstract}

\section{Introduction}

Cancer pain is a chronic pain with a unique and complex mechanism. At the early stage, cancer pain is mainly sensitized by primary afferent nerves caused by a series of paincausing substances released by tumor cells and inflammatory cells and continuously activated osteoclasts, while at the later stage, cancer pain is mainly caused by nerve compression and injury caused by tumor growth [1]. In recent years, with the aging of the society and the increasing incidence of cancer, about half of the patients with malignant tumors have different degrees of cancer pain, especially the incidence of cancer pain in advanced patients is as high as $60-80 \%$, which seriously affects the patients' disease recovery and quality of life [2]. Cancerous pain is a kind of pain with areas in need of repair or adjustment after the information transmission to the nerve center of feeling, although the clinical use of "three steps" analgesia method can partly control or relieve patients' pain, but as the disease progresses, the tumor load increases, and the increase of the 
analgesic drug resistance and adverse drug reactions are led to the decrease of the tolerance [3-5].

The understanding of pain in Huangdi Neijing is as follows: "The meridians are not only popular, but also endless around; the cold enters the meridians but delays, and the weeping fails; the blood is little outside the veins, and the qi is blocked in the middle, so the death is painful." Chinese medicine research believes that cancer pain is cancer toxin cohesion, meridian block blockage, and not general pain. If the human body is in a state of qi deficiency, it can not nourish the viscera and meridians, so pain will occur. Pain and its treatment are the diseases that are first felt and recognized by human beings. In other words, after the body is stimulated by the internal and external environment, pain-causing substances are generated and released from the tissues, pain receptors are sensitized, pain information is transmitted, sensory center perceives, and finally, pain enters the consciousness stage, which leads to pain [6]. Among them, pain-causing substances include bradykinin, histamine, 5-HT, and prostaglandin, which can excite or sensitize the receptors when they are stimulated by various stimuli [7]. At present, western medicine is widely used in the treatment of various kinds of pain, which has the effect of addiction and tolerance, which limits the clinical application and reduces the therapeutic effect $[8,9]$. The application of traditional Chinese medicine in the treatment of pain has a history of thousands of years. In ancient books and modern scientific research, it has been proved that more than 800 kinds of traditional Chinese medicine can effectively relieve pain $[10,11]$. Traditional Chinese medicine compound has the characteristics of multiherbs, multicomponents, and multitarget coordination, but the current research methods cannot fully explain its effects. Network pharmacology, from a macroscopic and systematic perspective, explores the overall effect of drug treatment on diseases, breaking the previous research mode of single compound and single target, and providing a new idea for the study of the TCM compound. There are 11 herbal medicines of plant origin, including Astragalus membranaceus, Poria cocos, honeysuckle, Cyperus, Paeonia lactiflora, bupleurum, Hedyotis diffusa, Scutellaria barbata, Trichosanthes kirilowii, Arisaema, and Scutellaria baicalensis. Among them, Astragalus membranaceus, Poria cocos, Cyperus, Paeonia lactiflora, and Scutellaria baicalensis have been used many times in the clinical research of pain treatment. However, the specific mechanism of pain relief by botanical drugs is still unclear. In order to optimize the clinical therapeutic effect of botanical drugs and clarify its mechanism of action, we obtained the effective chemical components and corresponding targets through TCMSP, ETCM, TCMID, and other databases; screened the painrelated targets from GeneCards, OMIM, DrugBank, and other databases; and constructed the "humanized" model by using Cytoscape software and STRING database. To screen the key components and key targets of therapeutic effect, GO and KEGG pathway enrichment analysis was carried out on the key targets through the DAVID platform, and the potential mechanism of 11 herbal extracts was obtained [12-18].

\section{Materials and Methods}

2.1. Identification and ADME Screening of Candidate Compounds of Traditional Chinese Medicine. In this study, all the active ingredients of 11 herbal medicines of botanical origin were searched by TCMSP, ETCM, and TCMID [19, 20]. The names of 11 traditional Chinese medicines, Astragalus membranaceus, Poria cocos, Honeysuicerae lonicerae, Radix Paeoniae lactiflora, Radix Bupleurum, Hedyotis diffusa, Scutellaria scleroides, Fructus Trichosanthis, Araceae aratidae, Radix Scutellariae, were input, and the main active components were further screened according to the oral availability $(\mathrm{OB}) \geq 30 \%$ and drug-like properties $(\mathrm{DL}) \geq 0.18$. $\mathrm{OB}$ and $\mathrm{DL}$ are the key indicators to evaluate the effective availability of drugs. Generally speaking, active ingredients with $\mathrm{OB} \geq 30 \%$ and $\mathrm{DL} \geq 0.18$ can be regarded as the main active ingredients of drugs [21].

\subsection{Prediction of Related Targets of Active Compounds in} Traditional Chinese Medicine. TCMSP, PharmMapper, SwissTarget, and other databases were used to screen the protein targets corresponding to the main active components of the abovementioned traditional Chinese medicine [22]. By using the retrieval function of UniproKBT in the UniProt database [23], all target gene names were corrected into official gene symbol names by inputting protein names, and active ingredients without targets were removed to obtain the information of active ingredients and related targets.

\subsection{Cancer Pain Disease Targets and Protein Collection.} According to DrugBank, GeneCards, and OMIM databases, the English "cancer pain" corresponding to pain was used as the key word to search and screen the related targets, and the targets of three disease databases were combined to remove the repetitive base, so as to obtain the potential targets of pain, and by using the retrieval function of UniProKBT in the UniProt database and by inputting the name of protein and limiting the species to human, all the potential targets of pain were identified. The name of target gene was corrected to official gene symbol, and the active components without target were eliminated to obtain the final disease target $[24,25]$.

2.4. Construction of "Compound-Target-Cancerous Pain" Signaling Pathway. The protein-protein interaction (PPI) network of potential targets and disease targets was constructed by using Cytoscape 3.7.2, and the two network graphs were fused and the intersection network was extracted by using the correlation function in the software. The direct and indirect target regulatory networks of 14 herbal medicines for cancer pain relief were obtained.

2.5. GO and KEGG Analysis. In order to clarify the potential action targets of active ingredients in 14 herbal medicines of plant origin, as well as the role of cancer-related pain targets in gene function and signaling pathway, GO and KEGG 
enrichment analysis was conducted in the DAVID database and Metascape platform based on annotation and visualization modules [26]. In this study, $R$ language programming was used for GO and KEGG pathway analysis, and set thresholds $P<0.05$. The possible mechanism of analgesic effects of 14 herbal herbs derived from plants was predicted through gene enrichment analysis, and the results were imported into the mapping software for visual mapping [27] (Table 1).

\section{Results}

3.1. Analysis of Components and ADME of Botanical Drugs. By searching TCMSP, ETCM, and TCMID databases and analyzing $\mathrm{ADME}$, based on $\mathrm{OB} \geq 30 \%$ and $\mathrm{DL} \geq 0.18$, a total of 154 active components were obtained from 11 kinds of herbal medicines, including 23 Astragalus membranaceus, 20 Poria cocos, 26 Honeysuckle, 18 Cyperus, 13 Paeonia alba, 17 Bupleurum, 7 Hedyotis diffusa, 29 Scutellaria barbata, 11 Trichosanthes kirilowii, 7 Arisaema, and 36 Scutellaria baicalensis. The compound information of 11 Traditional Chinese medicines is shown in Figure 1 and Table 2-12 [28].

\subsection{Target Prediction of Active Components in Traditional} Chinese Medicine. Through TCMSP, PharmMapper, SwissTarget database retrieval, and comparison with the UniProt database, a total of 341 protein targets of 153 active components of botanical drugs were obtained, including 247 Astragalus, 94 Poria cocos, 262 Honeysuckle, 178 Cyperus, 72 Paeonia lactiflora, 148 Hupleurum, 153 Hedyotis diffusa, 178 Scutellaria barbata, 9 Trichosanthes kirilowii, 39 Arisaema, and 90 Scutellaria baicalensis. The protein target information of 11 active components of traditional Chinese medicine is shown in Figure 2.

3.3. Screening of Pain-Related Targets. Based on OMIM, GeneCards, and DrugBank databases, pain disease-related targets were screened with "cancer pain" as the key word. The results showed that there were 670 pain disease-related targets in the OMIM database, 1086 GeneCards database, and 215 DrugBank database. A total of 1695 targets were obtained from the three databases. Compared with the UniProt database, 1110 verified human pain disease-related targets were obtained. By matching 341 protein targets of active components of plant-derived drugs, 141 common targets of plant-derived drugs and cancer pain diseases were obtained. The Venn diagram and detailed target information are shown in Figure 3 and Table 13, respectively.

3.4. PPI Network Construction. The interaction network of 141 potential targets was constructed by using the STRING database platform. The minimum interaction threshold was set to "0.9," and the results are shown in Figure 4. The nodes in the figure are intersection genes, and the edges represent the association degree of the intersection genes, and the thickness represents the binding degree. As shown in the figure, there
TABle 1: Website of database and network platform.

\begin{tabular}{lc}
\hline TCMSP & https://tcmspw.com/tcmsp.php \\
ETCM & http://www.tcmip.cn/ETCM/index.php/ \\
TCMID & http://www.Megabionet.org/tcmid/ \\
PharmMapper & http://lilab-ecust.cn/pharmmapper/ \\
SwissTarget & http://new.swisstargetprediction.ch/ \\
UniProt & https://www.uniprot.org/ \\
DrugBank & https://omim.org/ \\
GeneCards & https://omim.org/ \\
OMIM & https://omim.org/ \\
DAVID & https://metascape.org/gp/ \\
Metascape & https://metascape.org/gp/ \\
STRING & https://string-db.org/ \\
\hline
\end{tabular}

are 140 nodes and 538 edges. The PPI network diagram is imported into the software Cytoscape 3.7.2, and the network pictures are analyzed by tools. We selected those targets with greater than average degree and betweenness centrality, a total of 19 targets, as potential key targets for plant-derived drugs to exert analgesic effects The main proteins closely related to analgesic effect of botanical drugs are TNF, mapk1, Jun, and IL-6, as shown in Figure 5 and Table 14.

3.5. Construction of "Compound Traditional Chinese Medicine Compound Target Disease" Network Diagram of Analgesic Effect of Botanical Drugs. Nineteen core targets of botanical drug analgesia were obtained from 2.4, and the corresponding compounds and traditional Chinese medicine were screened and matched. The network diagram of "compound traditional Chinese medicine compound target disease" of botanical drug analgesia was predicted by using Cytoscape 3.7.2 software. The results are shown in Figures 6 and 7.

3.6. GO and KEGG Enrichment Analysis. Through DAVID and STRING databases, 141 targets of botanical drug pain disease were enriched and analyzed, including biological process (BP), molecular function (MF), and cell composition (CC). There are 193 biological processes, 47 molecular functions, and 22 cell components; 20 enriched genes are selected and mapped through the mapping software, as shown in Figure 8; 118 signaling pathways are involved in KEGG pathway abdominal muscle analysis, and 20 enriched genes are screened according to the number of enriched genes, as shown in Figure 9.

\section{Discussion}

Modern medicine believes that cancer pain is mainly caused by the disease itself or the pain caused in the treatment process. Tumors infiltrate bone tissue, and bone tissue destruction results in the release of prostaglandins. It invades the viscera, causing vasospasm, occlusion, and eventually necrosis of the viscera. Or it may compress peripheral nerves, nerve roots, spinal cord, etc. The patient's own mental tension and psychological pressure can cause pain disorders. There is no name for cancer pain in Chinese medicine, but according to the pain of different parts of 


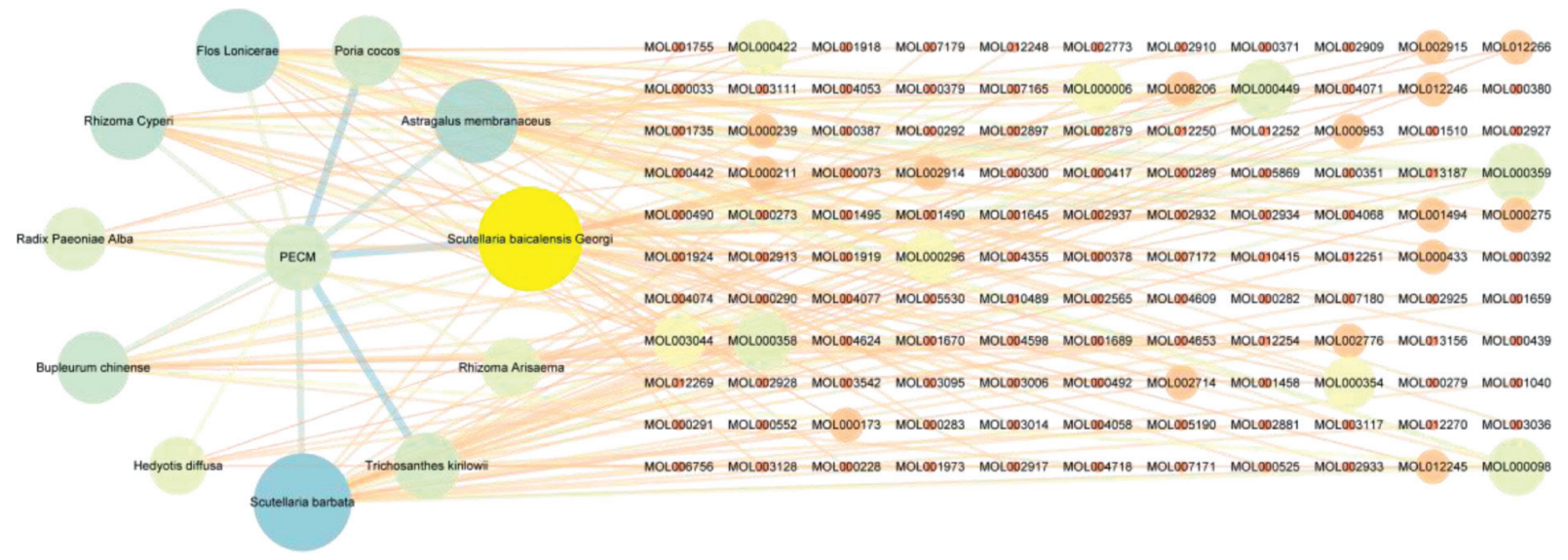

FIgURE 1: Network of botanical drugs, traditional Chinese medicine, and compounds.

TABLE 2: Related information of active components of Astragalus membranaceus.

\begin{tabular}{|c|c|c|c|}
\hline Mol ID & Molecule name & $\mathrm{OB}(\%)$ & DL \\
\hline MOL000211 & Mairin & 55.38 & 0.78 \\
\hline MOL000239 & Jaranol & 50.83 & 0.29 \\
\hline MOL000296 & Hederagenin & 36.91 & 0.75 \\
\hline MOL000033 & 24-Propylcholesterol & 36.23 & 0.78 \\
\hline MOL000354 & Isorhamnetin & 49.60 & 0.31 \\
\hline MOL000371 & 3,9-Di-O-methylnissolin & 53.74 & 0.48 \\
\hline MOL000374 & $5^{\prime}$-Hydroxyiso-muronulatol-2' $5^{\prime}$-di-O-glucoside & 41.72 & 0.69 \\
\hline MOL000378 & 7-O-Methylisomucronulatol & 74.69 & 0.30 \\
\hline MOL000379 & 9,10-Dimethoxypterocarpan-3-O- $\beta$-D-glucoside & 36.74 & 0.92 \\
\hline MOL000380 & (6aR,1laR)-9,10-Dimethoxy-6a,11a-dihydro-6H-benzofurano[ $[2,3]$-c]chromen-3-ol & 64.26 & 0.42 \\
\hline MOL000387 & Bifendate & 31.10 & 0.67 \\
\hline MOL000392 & Formononetin & 69.67 & 0.21 \\
\hline MOL000398 & Isoflavanone & 109.99 & 0.30 \\
\hline MOL000417 & Calycosin & 47.75 & 0.24 \\
\hline MOL000433 & FA & 68.96 & 0.71 \\
\hline MOL000438 & (3R)-3-(2-Hydroxy-3,4-dimethoxyphenyl)chroman-7-ol & 67.67 & 0.26 \\
\hline MOL000439 & Isomucronulatol-7,2'-di-O-glucosiole & 49.28 & 0.62 \\
\hline MOL000442 & 1,7-Dihydroxy-3,9-dimethoxy pterocarpene & 39.05 & 0.48 \\
\hline MOL000098 & Quercetin & 46.43 & 0.28 \\
\hline MOL000359 & Sitosterol & 36.91 & 0.75 \\
\hline MOL000433 & Folic acid & 68.96 & 0.71 \\
\hline MOL000239 & Kumatakenin & 50.83 & 0.29 \\
\hline MOL002565 & Medicarpin & 49.22 & 0.34 \\
\hline
\end{tabular}

cancer, it can be assigned to the pain syndrome of the corresponding parts. For example, pain caused by brain tumor, nasopharyngeal cancer, and cancer brain metastasis is classified as "headache." Cancer of the esophagus and lung can be classified as "chest pain." Pain caused by gastric cancer is classified as "stomachache." Pain caused by liver cancer is classified as "flank pain." Pain caused by pancreatic and colorectal cancer is classified as "abdominal pain." The pain caused by bone cancer and cancer bone metastasis is classified as "Bi syndrome" or "Gubi syndrome."

Pain is a kind of feeling of human disease caused by tissue injury, which stimulates the sensory system [29]. It is of great significance and research value in clinical diagnosis and treatment. Usually, the clinical pain mainly comes from the noxious stimulation of internal and external conditions caused by disease or surgery, which not only includes the defense response of the human body to noxious stimulation, but also a clinical manifestation of a variety of diseases and postoperative reactions [30]. Most of the patients have severe pain or chronic long-term pain and need to be treated with analgesic drugs. Cancer pain usually accompanies the whole course of cancer patients, so that patients are in pain, anxiety and even depression, and other negative emotions for a long time, causing some patients to lose confidence in life, which seriously affects the quality of life of patients. Therefore, how to effectively control the degree of cancer pain of cancer patients has great significance for the treatment of cancer and the improvement of life quality of cancer patients $[31,32]$. We need to understand the meaning of this. Therefore, the research of analgesic drugs has become one of the hot directions of modern drug research, but the clinical use of chemical drugs for acute or chronic pain, such as opioids and nonopioids: morphine, codeine, pethidine, aspirin, indomethacin, ibuprofen, and so on [33]. Although 
TABLE 3: Related information of active components of Poria cocos.

\begin{tabular}{|c|c|c|c|}
\hline Mol ID & Molecule name & $\begin{array}{l}\mathrm{OB} \\
(\%)\end{array}$ & $\mathrm{DL}$ \\
\hline MOL000273 & $\begin{array}{l}\text { (2R)-2-[(3S,5R,10S,13R,14R,16R,17R)-3,16-Dihydroxy-4,4,10,13,14-pentamethyl-2,3,5,6,12,15,16,17- } \\
\text { octahydro-1H-cyclopenta[a]phenanthren-17-yl]-6-methylhept-5-enoic acid }\end{array}$ & 30.93 & 0.81 \\
\hline MOL000275 & Trametenolic acid & 38.71 & 0.80 \\
\hline MOL000276 & 7,9(11)-Dehydropachymic acid & 35.11 & 0.81 \\
\hline MOL000279 & Cerevisterol & 37.96 & 0.77 \\
\hline MOL000280 & Dehydrotumulosic acid & 31.07 & 0.82 \\
\hline MOL000282 & Ergosta-7,22E-dien-3beta-ol & 43.51 & 0.72 \\
\hline MOL000283 & Ergosterol peroxide & 40.36 & 0.81 \\
\hline MOL000285 & Polyporenic acid C & 38.26 & 0.82 \\
\hline MOL000287 & $3 \beta$-Hydroxy-24-methylene-8-lanostene-21-oic acid & 38.70 & 0.81 \\
\hline MOL000289 & Pachymic acid & 33.63 & 0.81 \\
\hline MOL000290 & Poricoic acid A & 30.61 & 0.76 \\
\hline MOL000291 & Poricoic acid B & 30.52 & 0.75 \\
\hline MOL000292 & Poricoic acid C & 38.15 & 0.75 \\
\hline MOL000296 & Hederagenin & 36.91 & 0.75 \\
\hline MOL000300 & Dehydroeburicoic acid & 44.17 & 0.83 \\
\hline MOL000289 & Pachymic acid & 33.63 & 0.81 \\
\hline MOL000290 & Poricoic acid A & 30.61 & 0.76 \\
\hline MOL000291 & Poricoic acid B & 30.52 & 0.75 \\
\hline MOL000292 & Poricoic acid C & 38.15 & 0.75 \\
\hline MOL000275 & Trametenolic acid & 38.71 & 0.80 \\
\hline
\end{tabular}

TABLE 4: Related information of active components of honeysuckle.

\begin{tabular}{|c|c|c|c|}
\hline Mol ID & Molecule name & $\begin{array}{l}\mathrm{OB} \\
(\%)\end{array}$ & DL \\
\hline MOL001494 & Mandenol & 42.00 & 0.19 \\
\hline MOL001495 & Ethyl linolenate & 46.10 & 0.20 \\
\hline MOL002707 & Phytofluene & 43.18 & 0.50 \\
\hline MOL002914 & Eriodyctiol (flavanone) & 41.35 & 0.24 \\
\hline MOL003006 & $\begin{array}{c}\text { (-)-(3R,8S,9R,9aS,10aS)-9-Ethenyl-8-(beta-D-glucopyranosyloxy)-2,3,9,9a,10,10a-hexahydro-5-oxo-5H,8H- } \\
\text { pyrano[ [3, 4]-d] oxazolo[ }[2,3] \text {-a]pyridine-3-carboxylic acid_qt }\end{array}$ & 87.47 & 0.23 \\
\hline MOL003014 & Secologanic dibutylacetal_qt & 53.65 & 0.29 \\
\hline MOL002773 & Beta-carotene & 37.18 & 0.58 \\
\hline MOL003036 & ZINC03978781 & 43.83 & 0.76 \\
\hline MOL003044 & Chryseriol & 35.85 & 0.27 \\
\hline MOL003059 & Kryptoxanthin & 47.25 & 0.57 \\
\hline MOL003062 & 4,5'-Retro-.beta.,.beta.-Carotene-3,3'-dione, $4^{\prime}, 5^{\prime}$-didehydro- & 31.22 & 0.55 \\
\hline MOL003095 & 5-Hydroxy-7-methoxy-2-(3,4,5-trimethoxyphenyl)chromone & 51.96 & 0.41 \\
\hline MOL003101 & 7-Epi-Vogeloside & 46.13 & 0.58 \\
\hline MOL003108 & Caeruloside C & 55.64 & 0.73 \\
\hline MOL003111 & Centauroside_qt & 55.79 & 0.50 \\
\hline MOL003117 & Ioniceracetalides B_qt & 61.19 & 0.19 \\
\hline MOL003124 & Xylostosidine & 43.17 & 0.64 \\
\hline MOL003128 & Dinethylsecologanoside & 48.46 & 0.48 \\
\hline MOL000358 & Beta-sitosterol & 36.91 & 0.75 \\
\hline MOL000422 & Kaempferol & 41.88 & 0.24 \\
\hline MOL000449 & Stigmasterol & 43.83 & 0.76 \\
\hline MOL000006 & Luteolin & 36.16 & 0.25 \\
\hline MOL000098 & Quercetin & 46.43 & 0.28 \\
\hline MOL000359 & Sitosterol & 36.91 & 0.75 \\
\hline MOL000296 & Hederagenin & 36.91 & 0.75 \\
\hline MOL003044 & Chrysoeriol & 35.85 & 0.27 \\
\hline
\end{tabular}

the above methods have achieved good clinical efficacy, the side effects are often more serious due to the continuous and large dose of use. Traditional Chinese medicine believes that pain is due to body deficiency, deficiency of healthy qi, and invasion of external pathogens, which eventually leads to heat toxin and internal stagnation, obstruction of meridians and collaterals, qi stagnation, and blood stasis. Herbal medicine is one of the most important methods of traditional Chinese medicine in treating cancer pain, and studies have shown that bitter cold herbal medicine has exact 
TABLE 5: Related information of active components of cyperus.

\begin{tabular}{|c|c|c|c|}
\hline Mol ID & Molecule name & OB $(\%)$ & DL \\
\hline MOL003044 & Chryseriol & 35.85 & 0.27 \\
\hline MOL000354 & Isorhamnetin & 49.60 & 0.31 \\
\hline MOL003542 & 8-Isopentenyl-kaempferol & 38.04 & 0.39 \\
\hline MOL000358 & Beta-sitosterol & 36.91 & 0.75 \\
\hline MOL000359 & Sitosterol & 36.91 & 0.75 \\
\hline MOL004027 & 1,4-Epoxy-16-hydroxyheneicos-1,3,12,14,18-pentaene & 45.10 & 0.24 \\
\hline MOL004053 & Isodalbergin & 35.45 & 0.20 \\
\hline MOL004058 & Khell & 33.19 & 0.19 \\
\hline MOL004059 & Khellol glucoside & 74.96 & 0.72 \\
\hline MOL010489 & Resivit & 30.84 & 0.27 \\
\hline MOL004068 & Rosenonolactone & 79.84 & 0.37 \\
\hline MOL004071 & Hyndarin & 73.94 & 0.64 \\
\hline MOL004074 & Stigmasterol glucoside_qt & 43.83 & 0.76 \\
\hline MOL004077 & Sugeonyl acetate & 45.08 & 0.20 \\
\hline MOL000422 & Kaempferol & 41.88 & 0.24 \\
\hline MOL000449 & Stigmasterol & 43.83 & 0.76 \\
\hline MOL000006 & Luteolin & 36.16 & 0.25 \\
\hline MOL000098 & Quercetin & 46.43 & 0.28 \\
\hline
\end{tabular}

TABLE 6: Related information of active components of Paeonia lactiflora.

\begin{tabular}{|c|c|c|c|}
\hline Mol ID & Molecule name & $\begin{array}{l}\text { OB } \\
(\%)\end{array}$ & DL \\
\hline MOL001910 & 11-Alpha,12-alpha-epoxy-3beta-23-dihydroxy-30-norolean-20-en-28,12beta-olide & 64.77 & 0.38 \\
\hline MOL001918 & Paeoniflorgenone & 87.59 & 0.37 \\
\hline MOL001919 & $\begin{array}{c}\text { (3S,5R,8R,9R,10S,14S)-3,17-Dihydroxy-4,4,8,10,14-pentamethyl-2,3,5,6,7,9-hexahydro-1H-cyclopenta[a] } \\
\text { phenanthrene-15,16-dione }\end{array}$ & 43.56 & 0.53 \\
\hline MOL001921 & Lactiflorin & 49.12 & 0.80 \\
\hline MOL001924 & Paeoniflorin & 53.87 & 0.79 \\
\hline MOL001925 & paeoniflorin_qt & 68.18 & 0.40 \\
\hline MOL001928 & albiflorin_qt & 66.64 & 0.33 \\
\hline MOL001930 & Benzoyl paeoniflorin & 31.27 & 0.75 \\
\hline MOL000211 & Mairin & 55.38 & 0.78 \\
\hline MOL000358 & Beta-sitosterol & 36.91 & 0.75 \\
\hline MOL000359 & Sitosterol & 36.91 & 0.75 \\
\hline MOL000422 & Kaempferol & 41.88 & 0.24 \\
\hline MOL000492 & $(+)$-Catechin & 54.83 & 0.24 \\
\hline
\end{tabular}

TABLE 7: Related information of active components of bupleurum.

\begin{tabular}{|c|c|c|c|}
\hline Mol ID & Molecule name & $\mathrm{OB}(\%)$ & $\mathrm{DL}$ \\
\hline MOL001645 & Linoleyl acetate & 42.10 & 0.20 \\
\hline MOL002776 & Baicalin & 40.12 & 0.75 \\
\hline MOL000449 & Stigmasterol & 43.83 & 0.76 \\
\hline MOL000354 & Isorhamnetin & 49.60 & 0.31 \\
\hline MOL000422 & Kaempferol & 41.88 & 0.24 \\
\hline MOL004598 & 3,5,6,7-Tetramethoxy-2-(3,4,5-trimethoxyphenyl)chromone & 31.97 & 0.59 \\
\hline MOL004609 & Areapillin & 48.96 & 0.41 \\
\hline MOL013187 & Cubebin & 57.13 & 0.64 \\
\hline MOL004624 & Longikaurin A & 47.72 & 0.53 \\
\hline MOL004628 & Octalupine & 47.82 & 0.28 \\
\hline MOL004644 & Sainfuran & 79.91 & 0.23 \\
\hline MOL004648 & Troxerutin & 31.60 & 0.28 \\
\hline MOL004653 & (+)-Anomalin & 46.06 & 0.66 \\
\hline MOL004702 & Saikosaponin c_qt & 30.50 & 0.63 \\
\hline MOL004718 & $\alpha$-Spinasterol & 42.98 & 0.76 \\
\hline MOL000490 & Petunidin & 30.05 & 0.31 \\
\hline MOL000098 & Quercetin & 46.43 & 0.28 \\
\hline
\end{tabular}


TABLE 8: Related information of active components of Hedyotis diffusa.

\begin{tabular}{lccc}
\hline Mol ID & Molecule name & OB (\%) & DL \\
\hline MOL001646 & 2,3-Dimethoxy-6-methyanthraquinone & 34.86 & 0.26 \\
MOL001659 & Poriferasterol & 43.83 & 0.76 \\
MOL001663 & Oleanolic acid & 32.03 & \\
MOL001670 & 2-Methoxy-3-methyl-9,10-anthraquinone & 37.83 & 0.76 \\
MOL000449 & Stigmasterol & 43.83 & 0.21 \\
MOL000358 & Beta-sitosterol & 36.91 & 0.76 \\
MOL000098 & Quercetin & 46.43 & 0.75 \\
\hline
\end{tabular}

TABLE 9: Related information of active components of Scutellaria barbata.

\begin{tabular}{|c|c|c|c|}
\hline Mol ID & Molecule name & $\mathrm{OB}(\%)$ & $\mathrm{DL}$ \\
\hline MOL001040 & (2R)-5,7-Dihydroxy-2-(4-hydroxyphenyl)chroman-4-one & 42.36 & 0.21 \\
\hline MOL012245 & $5,7,4^{\prime}$-Trihydroxy-6-methoxyflavanone & 36.63 & 0.27 \\
\hline MOL012246 & 5,7,4'-Trihydroxy-8-methoxyflavanone & 74.24 & 0.26 \\
\hline MOL012248 & 5-Hydroxy-7,8-dimethoxy-2-(4-methoxyphenyl)chromone & 65.82 & 0.33 \\
\hline MOL012250 & 7-Hydroxy-5,8-dimethoxy-2-phenyl-chromone & 43.72 & 0.25 \\
\hline MOL012251 & Chrysin-5-methylether & 37.27 & 0.20 \\
\hline MOL012252 & 9,19-Cyclolanost-24-en-3-ol & 38.69 & 0.78 \\
\hline MOL002776 & Baicalin & 40.12 & 0.75 \\
\hline MOL012254 & Campesterol & 37.58 & 0.71 \\
\hline MOL000953 & CLR & 37.87 & 0.68 \\
\hline MOL000358 & Beta-sitosterol & 36.91 & 0.75 \\
\hline MOL012266 & Rivularin & 37.94 & 0.37 \\
\hline MOL001973 & Sitosteryl acetate & 40.39 & 0.85 \\
\hline MOL012269 & Stigmasta-5,22-dien-3-ol-acetate & 46.44 & 0.86 \\
\hline MOL012270 & Stigmastan-3,5,22-triene & 45.03 & 0.71 \\
\hline MOL000449 & Stigmasterol & 43.83 & 0.76 \\
\hline MOL000173 & Wogonin & 30.68 & 0.23 \\
\hline MOL001735 & Dinatin & 30.97 & 0.27 \\
\hline MOL001755 & 24-Ethylcholest-4-en-3-one & 36.08 & 0.76 \\
\hline MOL002714 & Baicalein & 33.52 & 0.21 \\
\hline MOL002719 & 6-Hydroxynaringenin & 33.23 & 0.24 \\
\hline MOL002915 & Salvigenin & 49.07 & 0.33 \\
\hline MOL000351 & Rhamnazin & 47.14 & 0.34 \\
\hline MOL000359 & Sitosterol & 36.91 & 0.75 \\
\hline MOL005190 & Eriodictyol & 71.79 & 0.24 \\
\hline MOL005869 & Daucostero_qt & 36.91 & 0.75 \\
\hline MOL000006 & Luteolin & 36.16 & 0.25 \\
\hline MOL008206 & Moslosooflavone & 44.09 & 0.25 \\
\hline MOL000098 & Quercetin & 46.43 & 0.28 \\
\hline
\end{tabular}

antitumor effect, with less toxic and side effects and obvious effect $[34,35]$. The clinical analgesic effect of chemical drugs has been fully recognized, but for patients with chronic pain, long-term use will produce dependence, peptic ulcer, and other adverse reactions. Accordingly, with the development of traditional Chinese medicine industry, the research of natural plant extracts for pain treatment is gradually deepening [36, 37].

Botanical medicine is an important method for the treatment of cancer pain. This method should be highly valued by medical staff. External application of traditional Chinese medicine is directly administered to patients on the body surface of medical staff. Under the condition of absorption on the skin or mucous membrane surface of the patient, it can reach the pain site of the patient, produce good analgesic effect, and gradually reduce the side effects caused by oral drugs [38]. In this study, 11 plant-derived drugs widely used in clinical pain treatment were selected as follows:
Astragalus membranaceus, Poria cocos, Honeysuckle, Cyperus, Paeonia lactiflora, Bupleurum, Hedyotis diffusa, Scutellaria barbata, Trichosanthes kirilowii, Arisaema, and Scutellaria baicalensis. Among them, Astragalus is sweet in taste and mild in nature. It is mainly used for blood numbness of limbs and hemiplegia. Poria cocos is sweet and light, and has a mild nature. Honeysuckle has the characteristics of sweet-cold clearing, light aroma, and drooping, is good for clearing away heat and detoxification, and treats carbuncle sores and boils. Cyperus is pungent, relieves bitterness, and tastes sweet, and it is harmonious, smooth, and unbiased, and works well for soothing the liver, regulating qi, and relieving pain. Paeonia lactiflora has sour, sweet, and bitter taste, it is mild cold in nature, and it has the effect of softening the liver and relieving pain. Bupleurum is bitter and mildly cold, and aromatic, relieves diarrhea, and mainly treats flank pain, irregular menstruation, and dysmenorrhea. Hedyotis diffusa is bitter and cold to clear diarrhea, and sweet and cold to 
TABLE 10: Related information of active components of Trichosanthes kirilowii.

\begin{tabular}{|c|c|c|c|}
\hline Mol ID & Molecule name & $\mathrm{OB}(\%)$ & DL \\
\hline MOL001494 & Mandenol & 42.00 & 0.19 \\
\hline MOL002881 & Diosmetin & 31.14 & 0.27 \\
\hline MOL004355 & Spinasterol & 42.98 & 0.76 \\
\hline MOL005530 & Hydroxygenkwanin & 36.47 & 0.27 \\
\hline MOL006756 & Schottenol & 37.42 & 0.75 \\
\hline MOL007165 & $10 \alpha$-Cucurbita-5,24-diene- $3 \beta$-ol & 44.02 & 0.74 \\
\hline MOL007171 & 5-Dehydrokarounidiol & 30.23 & 0.77 \\
\hline MOL007172 & 7-Oxo-dihydrokaro-unidiol & 36.85 & 0.75 \\
\hline MOL007175 & Karounidiol 3-o-benzoate & 43.99 & 0.50 \\
\hline MOL007179 & Linolenic acid ethyl ester & 46.10 & 0.20 \\
\hline MOL007180 & Vitamin-e & 32.29 & 0.70 \\
\hline MOL013146 & $8,11,14$-Docosatrienoic acid, methyl ester & 43.23 & 0.30 \\
\hline MOL013156 & [(2R)-2-[[[(2R)-2-(Benzoylamino)-3-phenylpropanoyl]amino]methyl]-3-phenylpropyl] acetate & 38.88 & 0.56 \\
\hline MOL001510 & 24-Epicampesterol & 37.58 & 0.71 \\
\hline MOL000358 & Beta-sitosterol & 36.91 & 0.75 \\
\hline MOL000359 & Sitosterol & 36.91 & 0.75 \\
\hline MOL000449 & Stigmasterol & 43.83 & 0.76 \\
\hline MOL000953 & CLR & 37.87 & 0.68 \\
\hline
\end{tabular}

TABle 11: Related information of active components of Rhizoma Arisaema.

\begin{tabular}{|c|c|c|c|}
\hline Mol ID & Molecule name & OB $(\%)$ & DL \\
\hline MOL013146 & $8,11,14$-Docosatrienoic acid, methyl ester & 43.23 & 0.30 \\
\hline MOL013156 & [(2R)-2-[[[(2R)-2-(Benzoylamino)-3-phenylpropanoyl]amino]methyl]-3-phenylpropyl] acetate & 38.88 & 0.56 \\
\hline MOL001510 & 24-Epicampesterol & 37.58 & 0.71 \\
\hline MOL000358 & Beta-sitosterol & 36.91 & 0.75 \\
\hline MOL000359 & Sitosterol & 36.91 & 0.75 \\
\hline MOL000449 & Stigmasterol & 43.83 & 0.76 \\
\hline MOL000953 & CLR & 37.87 & 0.68 \\
\hline
\end{tabular}

infiltrate and benefit, clear away heat and toxins, dissipate carbuncle, treat sore carbuncle, sore throat, and intestinal carbuncle. Scutellaria barbata has a pungent flavor that can disperse, relieve bitter cold, and clear diarrhea, not only dissipate blood stasis and cool blood to stop bleeding, but also clear heat and water to relieve drenching and swelling, and it is good at treating sores and cancers. Trichosanthes kirilowii is sweet and cold lubricating, and has the effect of clearing lungs, moisturizing dryness and resolving phlegm, reducing swelling, and relieving lumps. Arisaema has bitterness, dryness, and strong medicinal power, and it is especially good at dispelling meridian wind and phlegm, not only drying dampness and reducing phlegm, but also dispelling wind and relieving spasm. Scutellaria baicalensis is bitter cold, clears venting and dryness, and can cool blood and stop bleeding $[39,40]$.

Based on the database of TCMSP, ETCM and TCMID, and $\mathrm{OB} \geq 30 \%$ and $\mathrm{DL} \geq 0.18,23$ Astragalus membranaceus, 20 Poria cocos, 26 Honeysuckle, 18 Cyperus, 13 Paeonia lactiflora, 17 Bupleurum, 7 Hedyotis diffusa, 29 Scutellaria barbata, 11 Trichosanthes kirilowii, 7 Arisaema, and 36 Scutellaria baicalensis were obtained, with a total of 154 active components. Through TCMSP, PharmMapper, SwissTarget database retrieval, and comparison with the UniProt database, 341 protein targets of plant-derived drugs were obtained. Through 1110 targets related to human pain diseases, 141 common targets of plant-derived drugs pain diseases were obtained. The interaction network of 141 potential targets was constructed by using the STRING database platform, and the PPI network diagram was imported into Cytoscape 3.7.2 in the software, the network images were analyzed by tools, and the 19 targets with degree and betweenness centrality $>15$ were the potential key targets for the analgesic effect of botanical drugs. The main proteins closely related to the analgesic effect of botanical drugs were TNF, MAPK1, JUN, IL-6, and so on. GO enrichment analysis by the DAVID platform showed that the mechanism of pain relief by botanical drugs mainly involved 193 biological processes, 47 molecular functions, and 22 cell components; 118 signaling pathways were obtained by the abdominal muscle analysis of the KEGG pathway. The main biological processes are as follows: RNA polymerase II promoter, transcriptional DNA template, inflammatory reaction, transcriptional positive regulatory DNA template, apoptosis process, immune reaction, active regulation of angiogenesis, regulation of cell proliferation, activation of cysteine-type endopeptidase activity, regulation of apoptosis process, steroid hormone-mediated signaling pathway, lipopolysaccharide-mediated signaling pathway, and sequence-specific DNA binding transcription, positive regulation of NF-kappa B transcription factor activity, negative regulation of cell proliferation, negative regulation of transcription, and so on. Cell components include nucleus, cytoplasm, extracellular space, extracellular exosomes, cytosol, organic components of plasma membrane, intracellular mitochondria, external plasma membrane, endoplasmic reticulum, cell surface, extracellular matrix, postsynaptic membrane, complex receptors, membrane rafts, neuronal projection, cell junction, nuclear chromatin, 
TABLE 12: Related information of active components of Scutellaria baicalensis Georgi.

\begin{tabular}{|c|c|c|c|}
\hline Mol ID & Molecule name & OB $(\%)$ & DL \\
\hline MOL001689 & Acacetin & 34.97 & 0.24 \\
\hline MOL000173 & Wogonin & 30.68 & 0.23 \\
\hline MOL000228 & (2R)-7-Hydroxy-5-methoxy-2-phenylchroman-4-one & 55.23 & 0.20 \\
\hline MOL002714 & Baicalein & 33.52 & 0.21 \\
\hline MOL002908 & 5,8,2'-Trihydroxy-7-methoxyflavone & 37.01 & 0.27 \\
\hline MOL002909 & 5,7,2,5-Tetrahydroxy-8,6-dimethoxyflavone & 33.82 & 0.45 \\
\hline MOL002910 & Carthamidin & 41.15 & 0.24 \\
\hline MOL002911 & $2,6,2^{\prime}, 4^{\prime}$-Tetrahydroxy- $6^{\prime}$-methoxychaleone & 69.04 & 0.22 \\
\hline MOL002913 & Dihydrobaicalin_qt & 40.04 & 0.21 \\
\hline MOL002914 & Eriodyctiol (flavanone) & 41.35 & 0.24 \\
\hline MOL002915 & Salvigenin & 49.07 & 0.33 \\
\hline MOL002917 & $5,2^{\prime}, 6^{\prime}$-Trihydroxy-7,8-dimethoxyflavone & 45.05 & 0.33 \\
\hline MOL002925 & $5,7,2^{\prime}, 6^{\prime}$-Tetrahydroxyflavone & 37.01 & 0.24 \\
\hline MOL002926 & Dihydrooroxylin A & 38.72 & 0.23 \\
\hline MOL002927 & Skullcapflavone II & 69.51 & 0.44 \\
\hline MOL002928 & Oroxylin a & 41.37 & 0.23 \\
\hline MOL002932 & Panicolin & 76.26 & 0.29 \\
\hline MOL002933 & $5,7,4^{\prime}$-Trihydroxy-8-methoxyflavone & 36.56 & 0.27 \\
\hline MOL002934 & Neobaicalein & 104.34 & 0.44 \\
\hline MOL002937 & Dihydrooroxylin & 66.06 & 0.23 \\
\hline MOL000358 & Beta-sitosterol & 36.91 & 0.75 \\
\hline MOL000359 & Sitosterol & 36.91 & 0.75 \\
\hline MOL000525 & Norwogonin & 39.40 & 0.21 \\
\hline MOL000552 & 5,2'-Dihydroxy-6,7,8-trimethoxyflavone & 31.71 & 0.35 \\
\hline MOL000073 & Ent-Epicatechin & 48.96 & 0.24 \\
\hline MOL000449 & Stigmasterol & 43.83 & 0.76 \\
\hline MOL001458 & Coptisine & 30.67 & 0.86 \\
\hline MOL001490 & Bis[(2S)-2-ethylhexyl] benzene-1,2-dicarboxylate & 43.59 & 0.35 \\
\hline MOL001506 & Supraene & 33.55 & 0.42 \\
\hline MOL002879 & Diop & 43.59 & 0.39 \\
\hline MOL002897 & Epiberberine & 43.09 & 0.78 \\
\hline MOL008206 & Moslosooflavone & 44.09 & 0.25 \\
\hline MOL010415 & 11,13-Eicosadienoic acid, methyl ester & 39.28 & 0.23 \\
\hline MOL012245 & 5,7,4'-Trihydroxy-6-methoxyflavanone & 36.63 & 0.27 \\
\hline MOL012246 & $5,7,4^{\prime}$-Trihydroxy-8-methoxyflavanone & 74.24 & 0.26 \\
\hline MOL012266 & Rivularin & 37.94 & 0.37 \\
\hline
\end{tabular}

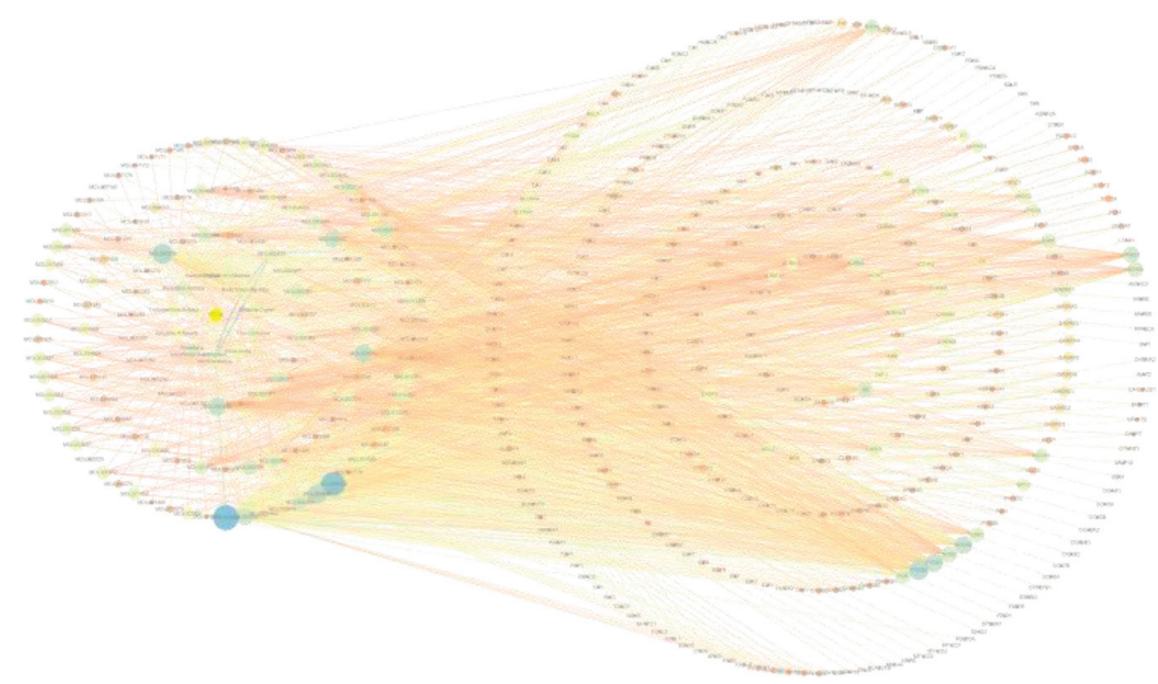

Figure 2: Network of traditional Chinese medicine active ingredients target. 


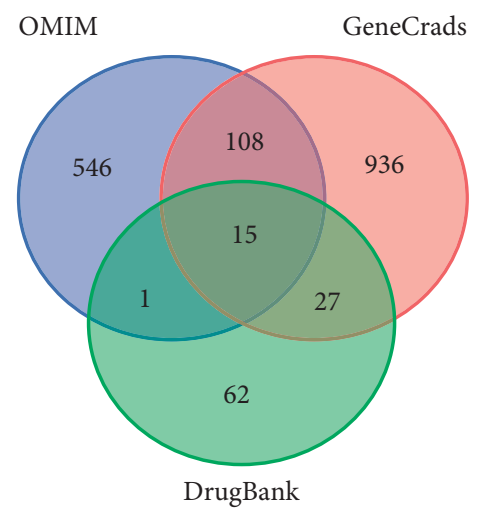

(a)

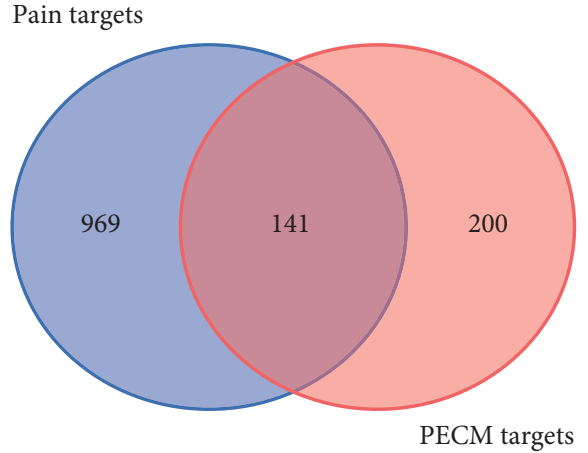

(b)

Figure 3: Venn map of target. ((a) is the Venn map of cancer pain-related targets in OMIM, GeneCards, and DrugBank databases; (b) is the Venn map of common targets of botanical drugs and cancer pain diseases).

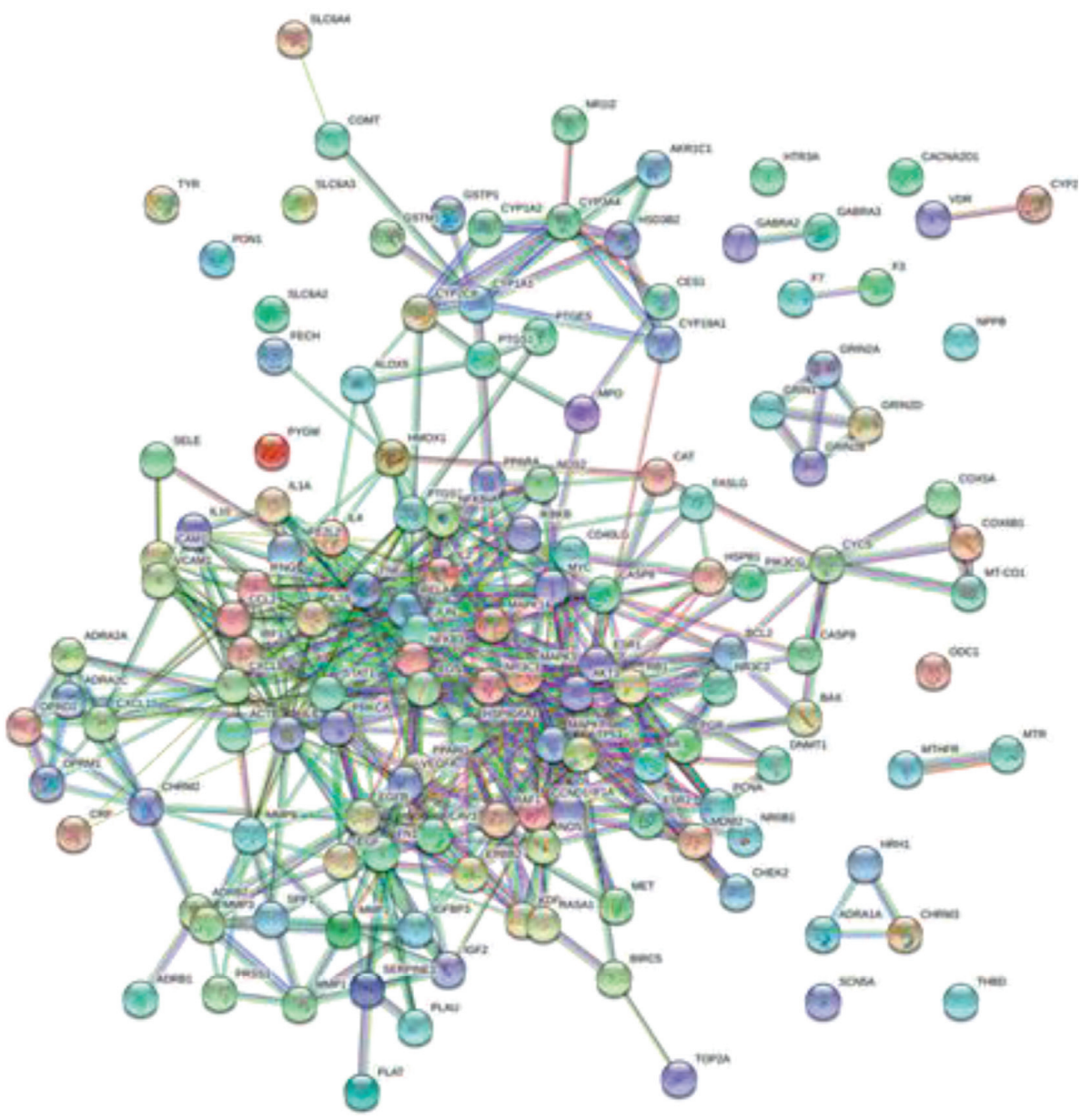

FIGURE 4: PPI network of botanical drugs cancer pain diseases.

spindle, RNA polymerase II transcription factor complexThings. Molecular functions include zinc ion binding, ATP binding transcription factor activity, DNA binding, heme node, cytokine activity, protein dimer activity, RNA polymerase II core promoter proximal region-specific sequence DNA binding, chromatin binding, steroid hormone receptor activity, growth factor activity, iron ion binding, transcriptional activator activity, steroid binding, transcriptional regulatory region DNA binding, amino acid endopeptidase activity, adrenaline binding, monooxygenase activity metalloendopeptidase activity, norepinephrine binding, aromatase activity, tumor necrosis factor receptor binding, MAPK activity, peroxidase activity, transmembrane receptor protein tyrosine kinase activity, core promoter sequence-specific DNA binding, drug binding, and 


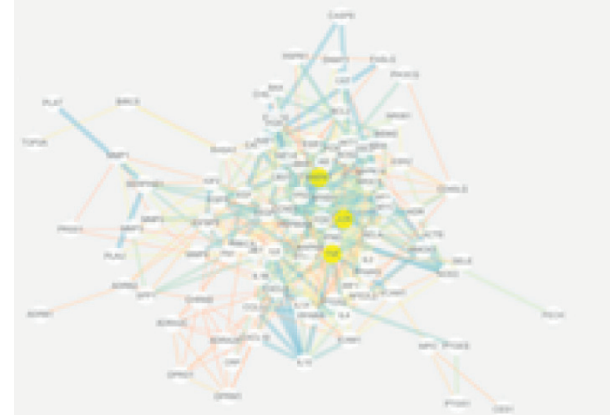

(a)

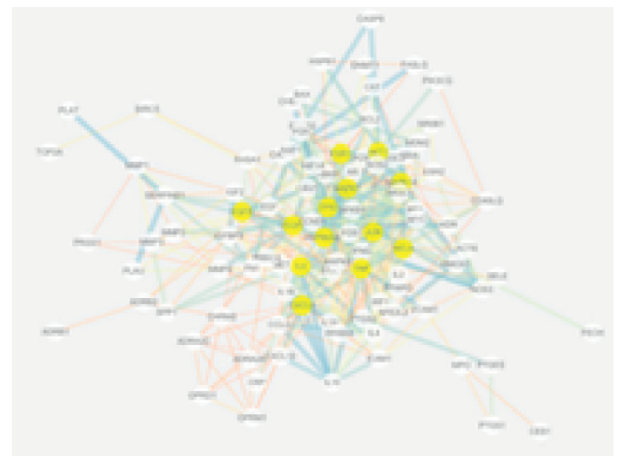

(c)

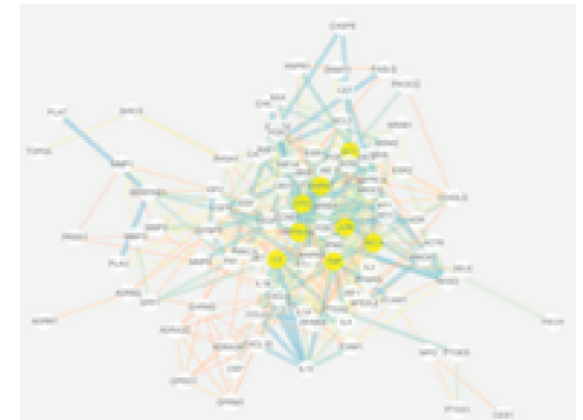

(b)

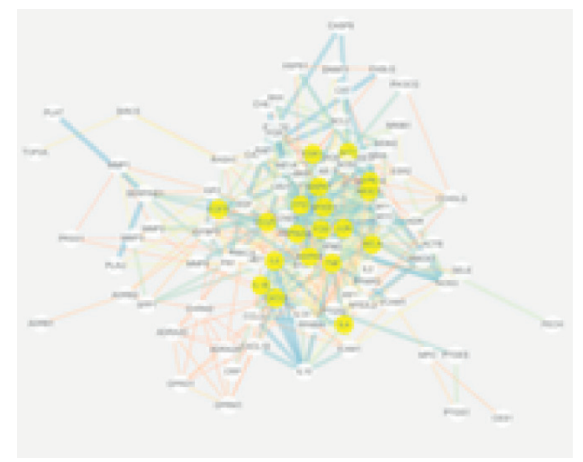

(d)

Figure 5: Core target map of different parameters of botanical drug cancer pain disease (selectivity and betweenness centrality are (a) 30; (b) 25; (c) 20; (d) 15).

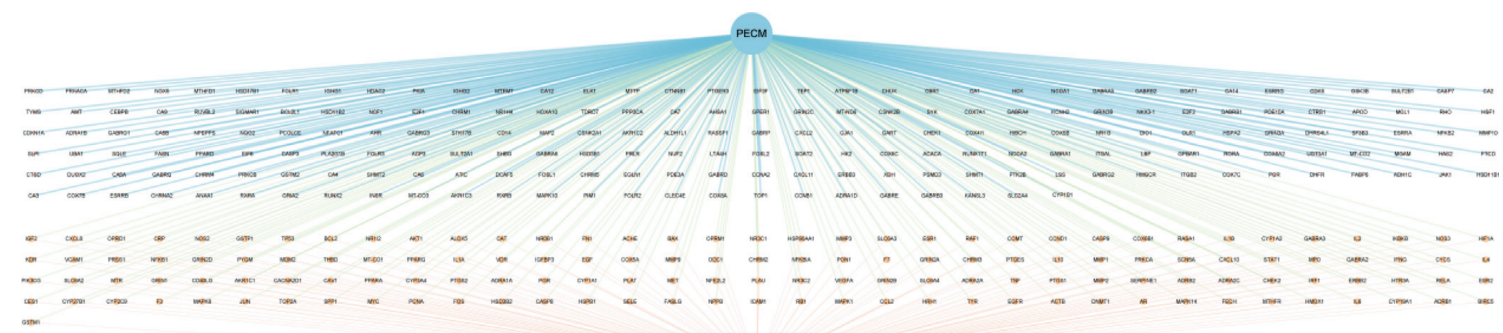

Figure 6: Traditional Chinese medicine target disease network.

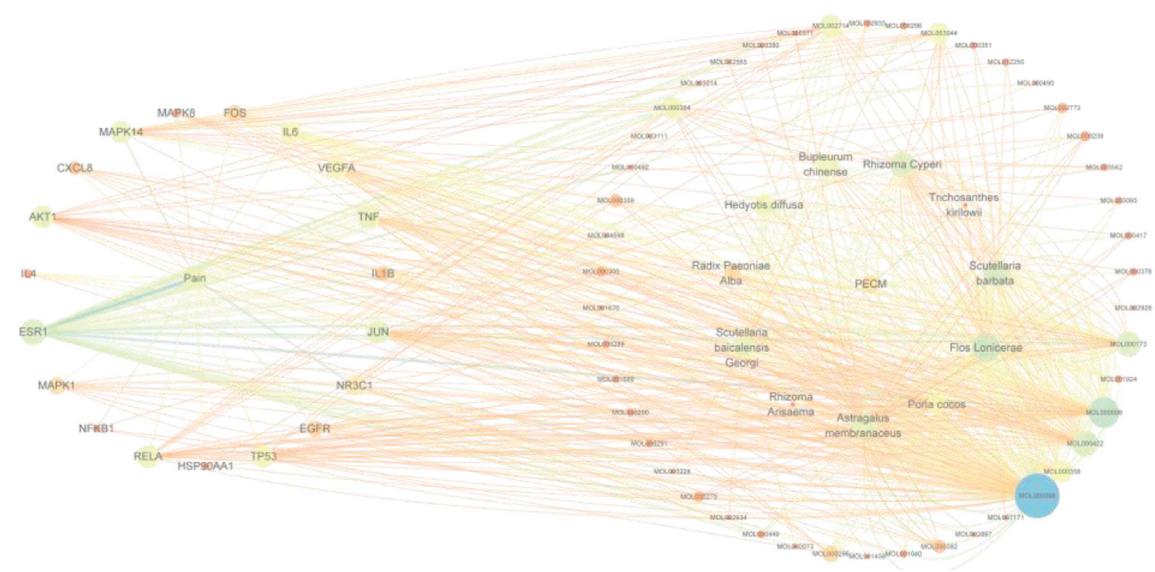

FIgURE 7: Compound Chinese medicine compound target disease network. 


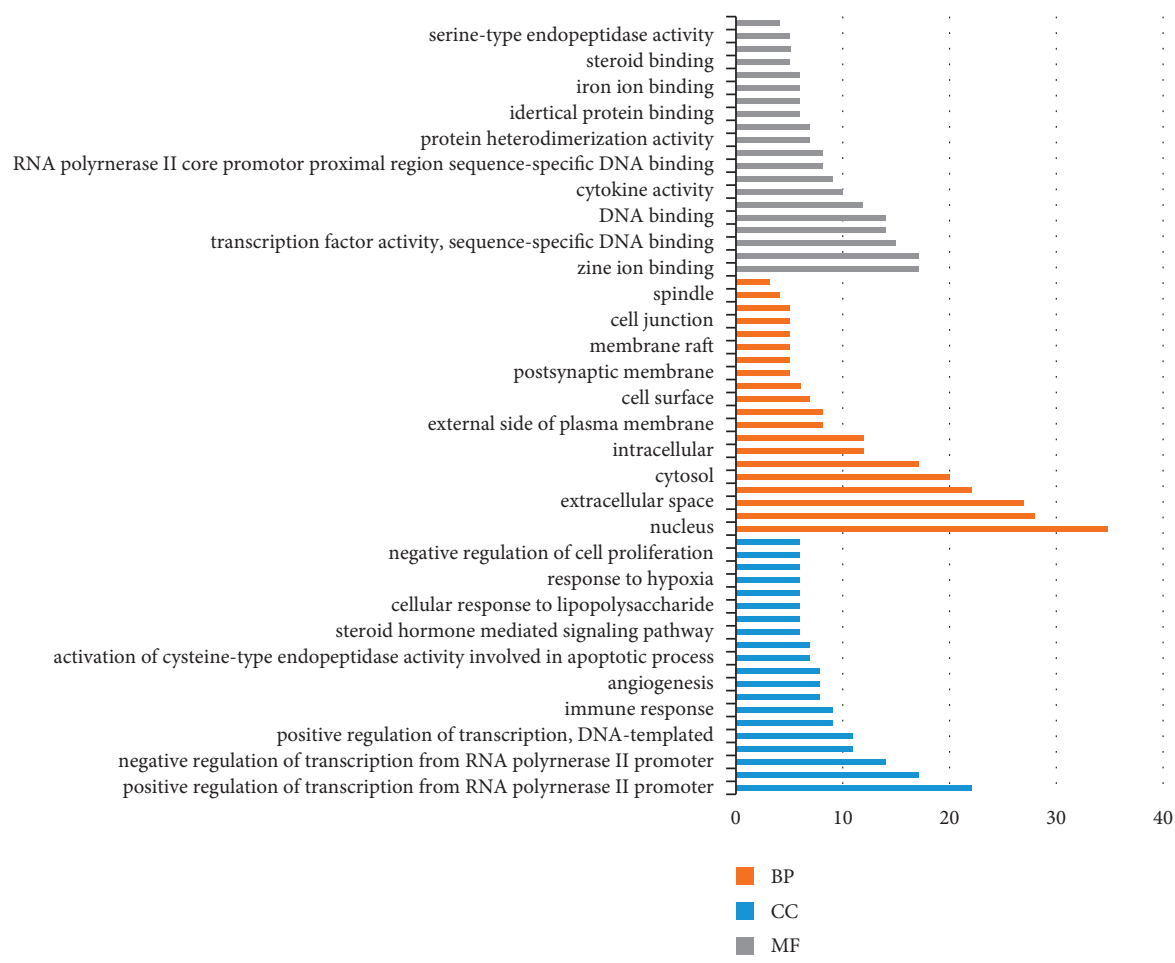

Figure 8: Enrichment analysis of GO in pain target of botanical drugs.

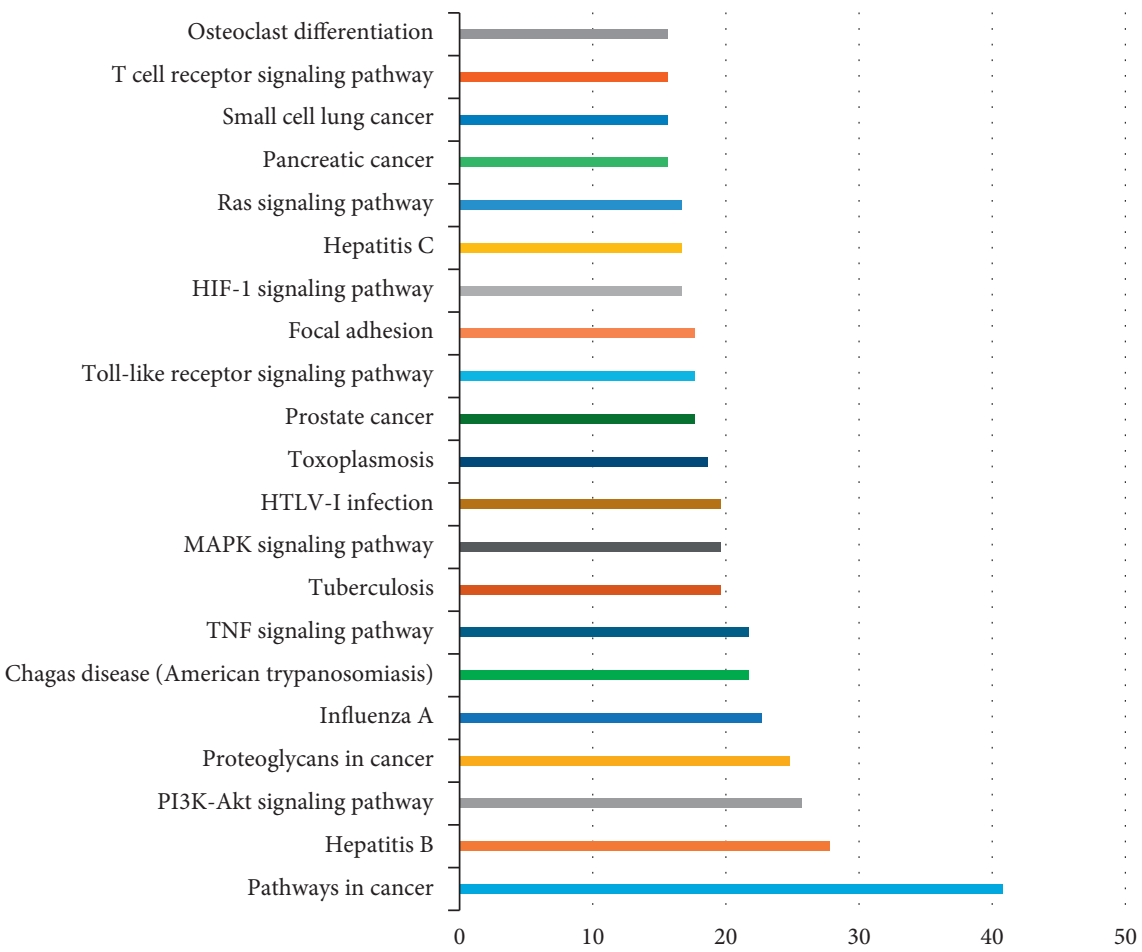

FIgURE 9: Enrichment analysis of the KEGG pathway, a pain target of botanical drugs.

chemokine activity. KEGG signaling pathways mainly include cancer, hepatitis B, PI3K-Akt signaling pathway, proteoglycan in cancer, influenza, tumor necrosis factor signaling pathway, pulmonary tuberculosis, MAPK signaling pathway, HTLV-I, toxoplasmosis infection, prostate cancer, Toll-like receptor signaling pathway, adhesive plaque, HIF-1 signaling pathway, hepatitis C, Ras signaling pathway, pancreatic cancer, small cell lung cancer, T-cell receptor signaling pathway, osteoclast differentiation, nonalcoholic fatty liver disease (NAFLD), herpes simplex infection, and cAMP signaling pathway ssc05206: 
TABLE 13: Common targets of botanical drugs and cancer pain diseases.

\begin{tabular}{|c|c|c|c|c|c|c|}
\hline \multicolumn{7}{|c|}{$141 \mathrm{common}$ target proteins } \\
\hline $\mathrm{ACHE}$ & CD40LG & EGFR & HRH1 & MAPK14 & NR1I2 & RAF1 \\
\hline ACTB & CES1 & ERBB2 & HSD3B2 & MAPK8 & NR3C1 & RASA1 \\
\hline ADRA1A & CHEK2 & ESR1 & HSP90AA1 & MDM2 & NR3C2 & $\mathrm{RB} 1$ \\
\hline ADRA2A & CHRM2 & ESR2 & HSPB1 & MET & ODC1 & RELA \\
\hline ADRA2C & CHRM3 & F3 & HTR3A & MMP1 & OPRD1 & SCN5A \\
\hline ADRB1 & COMT & F7 & ICAM1 & MMP2 & OPRM1 & SELE \\
\hline ADRB2 & COX5A & FASLG & IFNG & MMP3 & PCNA & SERPINE1 \\
\hline AKR1C1 & COX6B1 & FECH & IGF2 & MMP9 & PGR & SLC6A2 \\
\hline $\mathrm{AKT1}$ & CRP & FN1 & IGFBP3 & MPO & PIK3CG & SLC6A3 \\
\hline ALOX5 & CXCL10 & FOS & IKBKB & MT-CO1 & PLAT & SLC6A4 \\
\hline AR & CXCL8 & GABRA2 & IL10 & MTHFR & PLAU & SPP1 \\
\hline BAX & CYCS & GABRA3 & IL1A & MTR & PON1 & STAT1 \\
\hline BCL2 & CYP19A1 & GRIN1 & IL1B & MYC & PPARA & THBD \\
\hline BIRC5 & CYP1A1 & GRIN2A & IL2 & NFE2L2 & PPARG & TNF \\
\hline CACNA2D1 & CYP1A2 & GRIN2B & IL4 & NFKB1 & PRKCA & TOP2A \\
\hline CASP8 & CYP27B1 & GRIN2D & IL6 & NFKBIA & PRSS1 & TP53 \\
\hline CASP9 & СYP2C9 & GSTM1 & IRF1 & NOS2 & PTGES & TYR \\
\hline CAT & CYP3A4 & GSTP1 & JUN & NOS3 & PTGS1 & VCAM1 \\
\hline CAV1 & DNMT1 & HIF1A & KDR & NPPB & PTGS2 & VDR \\
\hline CCL2 & EGF & HMOX1 & MAPK1 & NR0B1 & PYGM & VEGFA \\
\hline CCND1 & & & & & & \\
\hline
\end{tabular}

TABLE 14: Core target map of different parameters of botanical drugs cancer pain disease.

\begin{tabular}{|c|c|c|c|c|c|c|}
\hline \multirow{2}{*}{$\frac{\text { Degree value } 30}{\text { TNF }}$} & \multirow{2}{*}{$\frac{\text { Degree value } 25}{\text { TNF }}$} & \multicolumn{2}{|c|}{ Degree value 20} & \multicolumn{3}{|c|}{ Degree value 15} \\
\hline & & TNF & CXCL8 & NR3C1 & MAPK1 & MAPK14 \\
\hline MAPK1 & IL6 & AKT1 & MAPK1 & IL1B & NFKB1 & IL4 \\
\hline \multirow{6}{*}{ JUN } & MAPK1 & RELA & EGFR & TNF & RELA & ESR1 \\
\hline & AKT1 & JUN & MAPK14 & IL6 & EGFR & HSP90AA1 \\
\hline & RELA & VEGFA & ESR1 & MAPK8 & JUN & ТР53 \\
\hline & JUN & TP53 & HSP90AA1 & CXCL8 & VEGFA & \\
\hline & HSP90AA1 & IL6 & & AKT1 & FOS & \\
\hline & TP53 & & & & & \\
\hline
\end{tabular}

microRNAs in tumors. It can be seen from the above signaling pathways that plant-derived drugs not only regulate the expression of inflammatory factors through inflammatory signaling pathways to relieve pain, but also actively participate in prostate cancer, pancreatic cancer, small cell lung cancer, and other cancer pathways, in order to achieve the effect of relieving cancer pain.

\section{Conclusion}

In conclusion, the material basis and the possible mechanism of action of the active components of plantderived drugs were preliminarily discussed through network pharmacology. In this article, the effective chemical components and corresponding targets of plantderived drugs were obtained by TCMSP, ETCM, TCMID, and other databases. GeneCards, OMIM, DrugBank, and other databases were used to screen out pain-related targets. Cytoscape software and STRING database were used to construct the "compound target" interaction network and protein interaction (PPI) network to screen the key components and key targets for therapeutic effects. GO and KEGG pathway enrichment analysis was performed on key targets through the DAVID platform, and the potential action mechanism of 11 herbal extracts of plant origin drugs was learned. We found 153 active ingredients from botanical drugs by TCMSP, ETCM, and TCMID databases, covering 341 protein targets in the human body. Combined with OMIM, GeneCards, and DrugBank databases, we excavated and matched 141 targets of plant-derived drugs and cancerous pain diseases. Through the analysis of the STRING platform and Cytoscape software, 19 core targets including TNF, MAPK1, JUN, and IL-6 were screened out. Go and KEGG enrichment showed that plant-derived drugs alleviated cancer pain processes involving 193 biological processes, 47 molecular functions, 22 cell components, and 118 signaling pathways. By screening genes involved in the KEGG signaling pathway, it was found that plant-derived drugs were mainly associated with PI3K-Akt signaling pathway, tumor necrosis factor signaling pathway, MAPK signaling pathway, Toll-like receptor signaling pathway, and HIF-1 signaling pathway in alleviating cancer pain. These results indicate that botanical drugs can positively affect the expression of inflammatory factors and apoptotic factors in the process of treatment and relief of cancer pain, which is expected to have a potential therapeutic effect on the relief of cancer pain. Although 
TCMSP and other databases were used to obtain the chemical components of plant-derived drugs, these data are not complete. In the later stage, UPLC, UPLC-Q-TOFMS, and other methods should be used to detect the chemical components of plant-derived drugs, in order to obtain more comprehensive chemical information to study the mechanism of action of traditional Chinese medicine monomer.

\section{Data Availability}

The datasets used and/or analyzed during the current study are available from the corresponding author on reasonable request.

\section{Conflicts of Interest}

The authors declare no conflicts of interest.

\section{References}

[1] O. Michelle and B. Andersen, "NCCN clinical practice guidelines in oncology (NCCN guidelines) distress management continue NCCN.org NCCN guidelines for patients," 2021, https://www.nccn.org/patientresources/patientresources.

[2] P. J. Christo and D. Mazloomdoost, "Cancer pain and analgesia," Annals of the New York Academy of Sciences, vol. 1138, no. 1, pp. 278-298, 2010.

[3] S. Gonella, R. Sperlinga, and V. Sciannameo, "Characteristics of breakthrough pain and its impact on quality of life in TerminallyIll cancer patients," Integrative Cancer Therapies, vol. 18, 2019.

[4] Q. Xu, Y. Zeng, W. Tang et al., "Multi-Task joint learning model for segmenting and classifying tongue images using a deep neural network," IEEE journal of biomedical and health informatics, vol. 24, no. 9, pp. 2481-2489, 2020.

[5] W. Tang, S. Wan, Z. Yang, A. E. Teschendorff, Q. Zou, and C. Sahinalp, "Tumor origin detection with tissue-specific miRNA and DNA methylation markers," Bioinformatics, vol. 34, no. 3, pp. 398-406, 2018.

[6] J. H. Zhao, J. F. Liu, and T. Y. Li, "Application effect and pain management status of comprehensive treatment in primary cancer patients," Journal of clinical rational drug use, vol. 10, no. 9, pp. 155-156, 2017.

[7] B. N. Nielsen, S. W. Henneberg, K. Schmiegelow, S. M. Friis, and J. Rømsing, "Peripherally applied opioids for postoperative pain: evidence of an analgesic effect? A systematic review and meta-analysis," Acta Anaesthesiologica Scandinavica, vol. 59, no. 7, pp. 830-845, 2015.

[8] Y. T. Zhao and L. R. Jia, "Application of traditional Chinese medicine in the treatment of cancer pain," China health nutrition, vol. 22, no. 4, pp. 232-234, 2012.

[9] S. Q. Liu, Y. Y. Zhou, and H. Y. Tu, "Comparison of traditional Chinese medicine and Western medicine in the treatment of chronic pain," Journal of Henan Medical College, vol. 28, no. 2, pp. 1058-1060, 2016.

[10] H. Zhang and C. H. Li, "Treatmeant of cancer pain with traditional Chinese medicine," Journal of clinical Chinese medicine, vol. 30, no. 4, pp. 758-760, 2018.

[11] X. T. Cui, H. Gao, Y. Z. Pan, Y. Q. Xing, and D. F. Yin, "Analgesic effect and safety of botanical drugs combined with morphine in cancer pain," Progress in Modern Biomedicine, vol. 18, no. 20, pp. 3883-3886, 2018.

[12] Y. Sun and J. Yang, "A bioinformatics investigation into the pharmacological mechanisms of the effect of Fufang Danshen on pain based on methodologies of network pharmacology," Scientific Reports, vol. 9, no. 1, p. 5913, 2019.

[13] R. Zhang, X. Zhu, H. Bai, and K. Ning, "Network pharmacology databases for traditional Chinese medicine: review and assessment," Frontiers in Pharmacology, vol. 10, p. 123, 2019.

[14] Q. Wu and Y. Hu, "Integrated network pharmacology and molecular docking strategy to explore the mechanism of medicinal and edible Astragali Radix-Atractylodis Macrocephalae Rhizoma acting on pneumonia via immunomodulation," Journal of Food Biochemistry, vol. 44, Article ID e13510, 2020.

[15] Y. Zou, H. Wu, X. Guo et al., "MK-FSVM-SVDD: a multiple kernel-based fuzzy SVM model for predicting DNA-binding proteins via support vector data description," Current Bioinformatics, vol. 16, no. 2, pp. 274-283, 2021.

[16] W. Zheng, L. Yin, X. Chen, Z. Ma, S. Liu, and B. Yang, "Knowledge base graph embedding module design for Visual question answering model," Pattern Recognition, vol. 120, Article ID 108153, 2021.

[17] W. Zheng, X. Liu, and L. Yin, "Research on image classification method based on improved multi-scale relational network," PeerJ Computer Science, vol. 7, p. e613, 2021.

[18] Z. Ma, W. Zheng, X. Chen, and L. Yin, "Joint embedding VQA model based on dynamic word vector," PeerJ Computer Science, vol. 7, p. e353, 2021.

[19] Y. J. Qu, R. R. Zhen, L. M. Zhang et al., "Uncovering the active compounds and effective mechanisms of the dried mature sarcocarp of Cornus officinalis Sieb. Et Zucc. For the treatment of Alzheimer's disease through a network pharmacology approach," BMC Complement Med Ther, vol. 20, no. 1, p. 157, 2020.

[20] X. Zhou, X. F. Zhang, D. Y. Guo et al., "Exploring the mechanism of lingzhu san in treating febrile seizures by using network pharmacology," Comb Chem High Throughput Screen, vol. 24, 2020.

[21] Y. Lu, J. Sun, M. Hu, X. Kong, W. Zhong, and C. Li, “Network pharmacology analysis to uncover the potential mechanisms of lycium barbarum on colorectal cancer," Interdiscip Sci, vol. 12, 2020.

[22] X. Q. Shi, S. J. Yue, Y. P. Tang et al., "A network pharmacology approach to investigate the blood enriching mechanism of Danggui buxue Decoction," Journal of Ethnopharmacology, vol. 235, pp. 227-242, 2019.

[23] T. H. Liu, W. H. Chen, X. D. Chen et al., "Network pharmacology identifies the mechanisms of action of TaohongSiwu decoction against essential hypertension," Medical Science Monitor, vol. 26, Article ID e920682, 2020.

[24] Y. Yu, G. Zhang, T. Han, and H. Huang, "Analysis of the pharmacological mechanism of Banxia Xiexin decoction in treating depression and ulcerative colitis based on a biological network module," BMC Complement Med Ther, vol. 20, no. 1, p. 199, 2020.

[25] M. Chu, M. S. Tsang, R. He, C. W. Lam, Z. B. Quan, and C. K. Wong, "The active compounds and therapeutic mechanisms of pentaherbs formula for oral and topical treatment of atopic dermatitis based on network pharmacology," Plants, vol. 9, no. 9, Article ID E1166, 2020.

[26] H. H. Xu, S. M. Li, R. Xu, L. Fang, H. Xu, and P. J. Tong, "Predication of the underlying mechanism of Bushenhuoxue formula acting on knee osteoarthritis via network pharmacology-based analyses combined with experimental 
validation," Journal of Ethnopharmacology, vol. 263, Article ID 113217, 2020.

[27] C. Zhang, Y. Liao, L. Liu et al., "A network pharmacology approach to investigate the active compounds and mechanisms of musk for ischemic stroke," Evid Based Complement Alternat Med, vol. 2020, Article ID 4063180, 14 pages, 2020.

[28] H. Yang, Y. Fan, J. Cheng et al., "Network pharmacologybased prediction of active ingredients and potential targets of shengdihuang decoction for treatment of dysfunctional uterine bleeding," Evid Based Complement Alternat Med, vol. 2020, Article ID 7370304, 12 pages, 2020.

[29] K. X. Du, D. Y. Li, J. Dong, J. Xu, and L. Ni, "Network pharmacology of frankincense myrrh in the treatment of cancer pain," World Journal of integrated traditional Chinese and Western medicine, vol. 15, no. 9, pp. 1591-1596, 2020.

[30] X. F. Zhang, L. Jia, H. Y. Xiao, and L. N. Zhang, "Progress in diagnosis and treatment of complex regional pain syndrome," Journal of Chinese PLA medical college, vol. 42, no. 7, pp. 8-12, 2020.

[31] L. Gao, Y. J. Yang, H. Y. Xu et al., “A randomized clinical trial of nerve block to manage end-stage pancreatic cancerous pain," Tumour Biol.vol. 35, no. 3, pp. 2297-2301, 2014.

[32] K. L. Gress, K. Charipova, A. D. Kaye, O. Viswanath, and I. Urits, "An overview of current recommendations and options for the management of cancer pain: a comprehensive review," Oncol Ther, vol. 8, 2020.

[33] S. Mathieson, C. G. Maher, G. E. Ferreira et al., "Deprescribing opioids in chronic non-cancer pain: systematic review of randomised trials," Drugs, vol. 80, no. 15, pp. 1563-1576, 2020.

[34] J. J. Shi and L. F. Yang, "Progress in diagnosis and treatment of cancer pain in traditional Chinese medicine," Clinical research of traditional Chinese medicine, vol. 10, no. 9, pp. 89-91, 2018.

[35] G. S. Zhu and W. J. Liu, "Analysis of understanding of cancer pain in TCM theory," Clinical research of TCM, vol. 8, no. 19, pp. 62-63, 2016.

[36] L. S. Branquinho, M. H. Verdan, E. D. Santos et al., "Aqueous extract from leaves of Doliocarpus dentatus (Aubl.) Standl. relieves pain without genotoxicity activity," Journal of Ethnopharmacology, vol. 266, Article ID 113440, 2020.

[37] H. Yu, H. Wang, T. Ma, A. Huang, Z. Lu, and X. Zhang, "TCM nonpharmacological interventions for chronic low-back pain: a protocol for systematic review and network meta-analysis," Medicine (Baltimore), vol. 99, no. 40, Article ID e22547, 2020.

[38] Y. Jiang, Study on Analgesic Mechanism of Botanical Drugs, Liaoning University of Traditional Chinese Medicine, Shenyang, China, 2012.

[39] F. S. Tian, Study On The Mechanism Of Compound Traditional Chinese Medicine Epidermal Analgesic, Tianjin Public Security Hospital, Tianjin, China, 2013.

[40] S. Tian, M. S. Miao, W. Z. Xiong, W. Xiong, M. Bai, and L. Li, "Analysis of external use function and application of Chinese Pharmacopoeia," Chinese Journal of experimental prescriptions, vol. 27, no. 8, pp. 161-167, 2021. 